Association for Information Systems AIS Electronic Library (AISeL)

Wirtschaftsinformatik Proceedings 2003

Wirtschaftsinformatik

September 2003

\title{
Ansätze zur marktorientierten Gestaltung webbasierter, akademischer Bildungsangebote
}

Gunnar Martin

Deutsches Forschungszentrum für Künstliche Intelligenz (DFKI), Saarbrücken, martin@iwi.uni-sb.de

August-Wilhelm Scheer

Deutsches Forschungszentrum für Künstliche Intelligenz (DFKI), Saarbrücken

Oliver Bohl

Universität Kassel

Udo Winand

Universität Kassel

Follow this and additional works at: http://aisel.aisnet.org/wi2003

\section{Recommended Citation}

Martin, Gunnar; Scheer, August-Wilhelm; Bohl, Oliver; and Winand, Udo, "Ansätze zur marktorientierten Gestaltung webbasierter, akademischer Bildungsangebote" (2003). Wirtschaftsinformatik Proceedings 2003. 88.

http://aisel.aisnet.org/wi2003/88

This material is brought to you by the Wirtschaftsinformatik at AIS Electronic Library (AISeL). It has been accepted for inclusion in Wirtschaftsinformatik Proceedings 2003 by an authorized administrator of AIS Electronic Library (AISeL). For more information, please contact elibrary@aisnet.org. 
In: Uhr, Wolfgang, Esswein, Werner \& Schoop, Eric (Hg.) 2003. Wirtschaftsinformatik 2003: Medien - Märkte - Mobilität, 2 Bde. Heidelberg: Physica-Verlag

ISBN: 3-7908-0111-9 (Band 1)

ISBN: 3-7908-0116-X (Band 2)

(C) Physica-Verlag Heidelberg 2003 


\title{
Ansätze zur marktorientierten Gestaltung web- basierter, akademischer Bildungsangebote
}

\author{
Gunnar Martin, August-Wilhelm Scheer \\ Deutsches Forschungszentrum für Künstliche Intelligenz (DFKI), Saarbrücken
}

\author{
Oliver Bohl, Udo Winand
}

Universität Kassel

Zusammenfassung: Die aktive Beteiligung deutscher Hochschulen auf dem Weiterbildungsmarkt ist trotz ihrer vielversprechenden Ausgangssituation nur gering ausgeprägt. Mit der Durchführung von eLearning-Forschungsprojekten besitzen Hochschulen jedoch eine gute Ausgangsposition auf dem Gebiet der marktorientierten und kooperativen Bildungsangebotserstellung. In diesem Beitrag werden aus Sicht des Forschungsprojektes „Bildungsnetzwerk WINFOLine“ Handlungsperspektiven beschrieben, die Hochschulen in die Lage versetzen, ihre Kompetenzen im Bereich der akademischen Ausbildung auf Bereiche der kommerziellen Aus- und Weiterbildung zu übertragen. Es werden Ansatzpunkte zur Neupositionierung und Profilbildung der Akteure auf dem Weiterbildungssektor am praktischen Beispiel aufgezeigt sowie Perspektiven zur marktorientierten, vernetzten Gestaltung web-basierter, akademischer Bildungsangebote aus Hochschulen heraus diskutiert.

Schlüsselworte: eLearning, Bildungsnetzwerk WINFOLine, Mass-Customization von Bildungsangeboten, Relationship-Marketing, Zielgruppenorientierung

\section{Marktorientierte Gestaltung akademischer Bildungsangebote - eine Herausforderung}

Im Rahmen der sich formierenden Wissensgesellschaft unterliegt das in Aus- und Weiterbildungsphasen erworbene Wissen immer durchdringenderen und schnelllebigeren Veränderungsprozessen [Krae 01, S. 191]. Hieraus resultieren LifelongLearning-Ansätzen, welche die Grenzen zwischen den einzelnen Phasen des Learning-Life-Cycles von der Erstausbildung bis zur Weiterbildung aufweichen. Nach dem Vorratslernprinzip erworbenes Wissen, welches über die Dauer eines Berufslebens kaum an Bedeutung verliert, gehört der Vergangenheit an [HuEn02, S. 545; Sche00, S. 182; Krae99, S. 18]. Die Aus- und Weiterbildung hat sich unlängst zu einem klassischen Markt entwickelt, auf dem Angebot und Nachfrage aufeinander 
treffen und Preise als Marktmechanismus fungieren. Diskontinuierliche Weiterbildungsphasen, auch im Anschluss an erste akademische Grade, entwickeln sich zu einem bedeutenden Bestandteil im Berufleben und spiegeln sich in der zunehmenden Gestaltung von unternehmerischen Personalentwicklungspfaden wider.

Diese Entwicklung bedeutet für Hochschulen, ,,dass ein Student nicht nur fünf oder sechs Jahre ein potenzieller Kunde für die Universitäten ist, sondern praktisch lebenslang, also 30-35 Jahre als Konsument von Aus- und Weiterbildungsmaßnahmen interessant ist" [Sche00, S. 182]. Wissen ist demzufolge als wirtschaftliches Gut und die Durchführung von wissensbildenden Lehrprozessen als Produkt bzw. Dienstleistung von Bildungsanbietern zu bezeichnen. Trotz ihrer starken Position im Bildungswesen, und speziell der akademischen Ausbildung, verhalten sich die deutschen staatlichen Hochschulen auf dem Wachstumsmarkt „Bildung“ besonders im Vergleich zu privaten US-Hochschulen zurückhaltend [HuEn02, S. 556ff]. Diese Beobachtung gilt besonders für den Bereich der Weiterbildung. obwohl sich durch das Vorhandensein bzw. das Entstehen von web-basierten, akademischen Bildungsangeboten eine erfolgsversprechende Basis für die Vermarktung und Mehrfachverwendung von Lehr-/Lerninhalten ergibt und sich Chancen zur Bedürfnisbefriedigung unterschiedlicher Bildungsszenarien bieten.

Eine Notwendigkeit zur Neuausrichtung dieser Strategie ergibt sich aber aus der Auffassung, dass zur nachhaltigen Sicherung der geschaffenen Angebote an deutschen Hochschulen eine Loslösung von Zuschüssen und kontinuierlichen Finanzströme unabdingbar ist. Eine Mehrfachverwendung und -vermarktung webbasierter Bildungsangebote, z.B. in Szenarien des zumindest finanziell lukrativ erscheinenden Sektors der betrieblichen Weiterbildung, aber auch ihr Austausch in und zwischen unterschiedlichen Organisationen der Aus- und Weiterbildung, wird oftmals vernachlässigt. Dies erstaunt, da sich ,der Eintritt in die betriebliche Weiterbildung eine Chance mehr [für Hochschulen bietet], über die Mehrfachnutzung ihrer Entwicklungen eine Refinanzierung zu erzielen. [Ehre ${ }^{+} 01$, S. 11].

In diesem Beitrag werden Basiselemente für die effiziente und effektive Gestaltung marktorientierter web-basierter, akademischer Bildungsangebote und dienstleistungen dargestellt. Dabei sind unter web-basierten akademischen Bildungsangeboten und -dienstleistungen Angebote zur Vermittlung wissenschaftlicher Inhalte zu verstehen, welche Internettechnologien zur Unterstützung bzw. Substitution von Präsenzphasen der Wissensvermittlung nutzen. In diesem Kontext erfolgt ausdrücklich keine Beschränkung auf die Hochschulen als Orte der Wissensvermittlung. Vielmehr werden aus Sicht des Forschungsprojektes „Bildungsnetzwerk WINFOLine“ Handlungsperspektiven beschrieben, die Hochschulen in die Lage versetzen, vorhandene Kompetenzen im Bereich der akademischen Ausbildung auf Bereiche der kommerziellen Aus- und Weiterbildung zu übertragen. Ferner werden Ansatzpunkte zur Neupositionierung und Profilbildung der staatlichen deutschen Hochschulen auf dem Weiterbildungssektor am praktischen Beispiel aufgezeigt. 


\section{Zielgruppenorientierte Gestaltung akademischer Bildungsangebote - ein Erfolgsfaktor}

In diesem Abschnitt wird dargestellt, inwiefern ein adäquates Relationship Marketing bei der Gestaltung web-basierter, akademischer Bildungsangebote einen Erfolgsfaktor darstellen kann. Unter Erfolgsfaktoren können Begriffe wie „Erfolgsdeterminanten“, „Erfolgspotenziale“ oder „Wettbewerbsvorteile“ gefasst werden. Allgemein lassen sich hierunter Ansätze und Methoden subsumieren, die in direktem Zusammenhang mit Unternehmenserfolgen stehen [Dell91, S. 241].

Als eine Voraussetzung für die Marktorientierung von Hochschulen und dem angestrebten Aufbau ökonomischer Wechselbarrieren ist die Kenntnis über potenzielle Zielgruppen sowie deren generelle Anspruchsgrundlagen unabdingbar. Besonders vor dem Hintergrund der Angebotspositionierung entlang aller Phasen des Learning-Life-Cycles erscheint dieses Vorgehen ratsam. Der Fokus liegt im Aufbau und der Etablierung schwer imitierbarer Kundenbeziehungen, aus denen im Falle eines Anbieterwechsels hohe Umstellungskosten für den Abnehmer resultieren [Port97, S. 33].

Innerhalb einer ersten undifferenzierten Betrachtung des Bildungsmarktes können die Segmente der privaten Nachfrager (Education-to-Customer) und der institutionellen Nachfrager (Education-to-Business; Education-to-Education) identifiziert werden. Trotz der Diffusität des gesetzten Zielgruppenclusters zeigen sich segmentübergreifende Ansprüche an den entgeltlichen Bezug der „Ware Bildung“. Laut Bentlage/Hummel bestehen diese vor allem in der Sicherstellung der Aktualität der Inhalte, der Individualität von Lernbeziehungen, der Just-inTime- Bereitstellung von Lerninhalten, der Reichhaltigkeit an Methodenwissen und der Bereitstellung integrierter Bildungsangebote und -programme [BeHu01, S. 148ff]. Ungeachtet der genannten Gemeinsamkeiten führen unterschiedliche Akzentsetzungen sowie, Kernkompetenzenund Motivationen des Leistungsanbieters in der Angebotsgestaltungsphase zum Entstehen von differenzierten Bildungsangeboten. Die entstehenden Bildungsangebote sind stark durch die Sicht des Leistungsanbieters geprägt und werden auf die potenziellen Kunden transferiert. Es wird unterstellt, dass das Angebot den Kundenbedürfnisse und strukturen weitgehend entspicht bzw. sich diese an das Bildungsangebot anpassen. Im Gegensatz hierzu entsteht für die marktorientierte Angebotsgestaltung die Notwendigkeit, nicht nur (Teil-) Funktionen im Leistungserstellungsprozess isoliert voreinander zu sehen, sondern die Tätigkeit vom Endergebnis her zu betrachten, also vom Standpunkt des Kunden aus [Druc95, S. 1]. Ziel ist es „die Bedürfnisse von Individuen oder Organisationen zu ergründen, entsprechende Güter [hier: als Bildungsangebote zu verstehen] bereitszustellen und durch den Austausch dieser Güter letztlich zur Bedürfnisbefreidigung beizutragen" [Hild98, S. 55]. Der beschriebene Ansatz der zielgruppengerechten Gestaltung von Bildungsangeboten kann auch als "Customizing the Relationship“ begriffen 
werden., Dieser ist durch das Vorhandensein der nachstehenden Bestandteile charakterisiert [Hild98, S. 58ff]:

1. Die Interaktion zwischen Bildungsanbietern und -nachfragern zielt auf die „explizite Nennung spezifischer Bedürfnisse, Vorschläge und Wünsche des Kunden und [andererseits] das active listening" [Hild98, S. 59] des Anbieters ab. Von besonderer Signifikanz sind Direktheit und Intensität der Interaktion sowie die gegenseitige Offenheit im Informationsverhalten. Die Kenntnis voneinander, sowie das Wohlwollen ggü. des voneinander Lernens, gilt als konstitutiv für das Funktionieren der Interaktionsbeziehung. Als Besonderheit bei institutionellen Beziehungen erweist sich die Festlegung eines gemeinsamen Zielspektrums der durchzuführenden Aktivitäten auf Anbieter- und Abnehmerseite als kritisch. Hierbei besteht die Herausforderung in der Realisierung in einem breiten Andocken beim Abnehmer auf verschiedenen Hierachieebenen und unterschiedlichen Funktionsbereichen. Demzufolge repräsentiert die Interaktion zwischen Bildungsanbietern und Bildungsnachfragern einen Kernbestandteil im Prozess der marktorientierten Bildungsangebotsgestaltung und ist eine wesentliche Vorstufe zur Integration der Nachfrager in den Leistungsentstehungsprozess.

2. Die Integration des Nachfragers kennzeichnet die Einbeziehung desselben entlang aller Phasen des Leistungsentstehungsprozesses. D. h. beginnend mit der Planung und Entwicklung, über die Umsetzung bis hin zur technologischen und organisatorischen Implementierung der Bildungsangebote und dienstleistungen. Auf die Gestaltung von Aus- und Weiterbildungsangeboten übertragen bedeutet dies, die Angebotsproduktions-, Konfigurations- und Customizingprozesse apriorisch mit der abnehmerseitigen Bildungsstrategie in Kooperation zwischen Abnehmern und Anbietern abzustimmen. Durch die Integration wird der Abnehmer zum Bestandteil der wertschöpfenden Tätigkeit und somit Co-Produzent innerhalb eines entstehenden Wertschöpfungsnetzwerkes. Der Erkenntnisgewinn aus dem wechselseitigen Informationsaustausch versetzt die Bildungsanbieter wiederum in die Lage, Kundenbedürfnisse und Bedürfnisstrukturen zu erkennen und Leistungen zu deren zielgerichteter Befriedigung optimiert bereitzustellen [GrMa02, S. 320]. Diesem Ansatz folgend werden in die Wertschöpfung integrierte Bildungsabnehmer zu langfristigen Kooperationspartnern der Hochschulen im Hinblick auf die Gestaltung, Entwicklung, Erprobung und Qualitätsförderung von Bildungsangeboten. Den Abnehmern kommt dabei besonders eine Indikatorfunktion bei der Identifikation von Veränderungsprozessen bzgl. der Bildungsnachfrage zu.

3. Die Individualisierung der Angebotsgestaltung bildet den Kern der marktorientierten Bildungsangebotsgestaltung. Die perspektivische Abkehr vom Massenprodukt „Bildung“ über Ansätze der Mass-Customization bis hin zur völligen Maßschneiderung von Bildungsoffertenwird möglich und richtet sich stets an den Wünschen potenzieller institutioneller Zielgruppen oder einzelner Kunden (Segment-of-One) aus. Das Ergebnis ist eine langfristige Veränderung von 
Bildungsangeboten hinsichtlich ihrer Qualität und Flexibilität. Zu beachten ist, dass die Angebotserstellung von vollständig individualisierten Bildungsleistungen für stark differenzierte (diffuse) Zielgruppen aus Effizienzgesichtspunkten kaum vertreten werden kann. Vielmehr kommen Aspekte der Mehrfachverwendung von Lehr-/Lerninhalten, Standardisierungsbestrebungen und Modularisierungsaspekte zum Tragen und stellen entscheidende Einflussfaktoren für die Effektivität und Effizienz der Individualisierung von web-basierten Bildungsangeboten und -dienstleistungen dar.

4. Die Selektion kennzeichnet die Auswahl und Fokussierung bestimmter Zielgruppen von Nachfragern nach Bildungsangeboten und -dienstleistungen. Nicht alle Nachfragersegmente weisen eine gleichartige Profitabilität auf. Es erscheint entscheidend, langfristig erfolgsversprechende Zielkundensegmente frühzeitig $\mathrm{zu}$ identifizieren und mit zielgruppenkonformen, integrierten Bildungsangeboten und -dienstleistungen zu versorgen [Riek95, S. 47ff]. Das Aufführen der Selektion als Bestandteil der zielgruppengerechten Gestaltung von Bildungsangeboten dient in diesem Kontext lediglich der formalen Vollständigkeit. Dieser Aspekt soll im Rahmen der weiterführenden Betrachtung nicht näher untersucht werden.

Wie anhand der dargelegten Einflussfaktoren aufgezeigt, erweist sich ein adäquates Marketing und Management der Transaktionsbeziehungen zwischen Anbietern und Abnehmern von Bildungsdienstleistungen als ein potenzieller Erfolgsfaktor. Unter Transaktionen sind in diesem Kontext alle Interaktionsprozesse zu subsumieren, bei denen ein Leistungsaustausch zwischen den Marktakteuren im Vordergrund des Handelns steht [Hild98, S. 55] Hierbei bieten Forschungsprojekte eine gute Ausgangsbasis für das Sammeln von Erfahrungen bei der zielgruppengerechten Ansprache unterschiedlicher Nachfragergruppen, bei der zielgruppenkonformen Planung und Umsetzung akademischer Bildungsangebote sowie bei deren Erprobung in verschiedenen Aus- und Weiterbildungsszenarien. Im Zeitraum der Forschungsförderung unterliegen die Projekte i.d.R. nicht dem Druck „Kunden aufzubauen oder geeignete Produkte zur Befriedigung der Nachfrage zu entwickeln“ [Port97, S. 277]. Der Gefahr des sogenannten kurzen Zeithorizonts, der Fehleinschätzung in der Behandlung von Problemen, die ,eher nach einer kurzfristigen Zweckmäßigkeit bewältigt werden, statt nach dem Ergebnis einer Analyse der zukünftigen Bedingungen“" [Port97, S. 278] zu suchen, kann entgegengewirkt werden. Ein Lösungsansatz zur Gestaltung und für den Vertrieb von zielgruppengerechten web-basierten, akademischen eLearning- Bildungsangeboten wird im Folgenden am Beispiel des Forschungsprojektes „Bildungsnetzwerk WINFOLine“" aufgezeigt und mit den seit 1997 gewonnenen Erfahrungen und Überlegungen in Kontext gesetzt. 


\section{WINFOLine - ein Lösungsansatz zur kooperativen, marktorientierten Gestaltung web-basierter, akademischer Bildungsangebote}

WINFOLine ist ein interuniversitäres und bundeslandübergreifendes Bildungsnetzwerk im Bereich der Wirtschaftsinformatik mit Partnern aus Hochschulen und Industrie. Neben einer erprobten Kooperationsarchitektur bildet ein Bildungsproduktpool, der multimediale Kursangebote und unterstützende Dienstleistungen beinhaltet, den Kern des Bildungsnetzwerks. Mit den auf dieser Basis aggregierten Bildungsangeboten und -dienstleistungen wendet sich WINFOLine an heterogene Abnehmergruppen in der Aus- und Weiterbildung, welche durch zielgruppenorientierte Anreizsysteme und die marktorientierte Gestaltung zur Abnahme der Bildungsangebote motiviert werden. WINFOLine kann auf mehr als 2.200 Studierende aus verschiedenen Bereichen der kommerziellen und nicht-kommerziellen Aus- und Weiterbildung verweisen.

Im Bildungsnetzwerk WINFOLine dient die konsequente Nutzung von Internettechnologien im Rahmen der Bildungsangebote (z.B. Web-based Trainings) und in den begleitenden Bildungsdienstleistungen (z.B. Online-Tutoring) als Unique Selling Proposition gegenüber etablierten, traditionellen Bildungsansätzen der Präsenz- und Fernlehre. Der Nutzen des gewählten elektronischen Lernens und Lehrens besteht in der zielgerichteten Angebotserteilung und dem Bezug von ortsund zeitpunktunabhängig organisierten Bildungsmaßnahmen im Sinne des Just-inTime- bzw. On-Demand Lernens [Wina ${ }^{+} 96$, S. 304]. Ferner realisiert das Bildungsnetzwerk ein dynamisches Studienangebot, das einen Beitrag zur Aktualitäts- und Qualitätssicherung der Lehre leistet, und den privaten wie organisationalen Abnehmern in diversen Bereichen der Aus- und Weiterbildung ein breites Spektrum an grundlegenden sowie vertiefenden Lehrveranstaltungen der Disziplin Wirtschaftsinformatik offeriert.

Im Folgenden werden die im zweiten Abschnitt identifizierten Bestandteile des zielgruppenorientierten Marketings und Managements von Kundenbeziehungen und ihre Abdeckung und Berücksichtigung im Bildungsnetzwerk WINFOLine diskutiert.

\subsection{Die Interaktions- und Integrationskomponenten im Angebotsgestaltungsprozess des Bildungsnetzwerks}

Ein bedeutendes Nutzenpotenzial des etablierten Bildungsangebots- und Bildungsdienstleistungpools ist in der Erhöhung der Auswahlmöglichkeiten leistungsbeziehender Partner zu sehen. Zu den anvisierten Abnehmergruppen des Bildungsnetzwerks WINFOLine zählen: 


\section{Education-to-Education (E2E)- Abnehmer:}

Abnehmer aus dem horizontalen E2E-Markt: Hochschulen oder weitere Bildungsanbieter, die über kein Wirtschaftsinformatik-Angebot verfügen und dieses mit Hilfe des Bildungsnetzwerkes WINFOLine neu aufbauen und ihr Lehrveranstaltungsportfolio erweitern wollen.

Abnehmer aus dem vertikalen E2E-Markt: Hochschulen oder weitere Bildungsanbieter, die über Studienangebote der Wirtschaftsinformatik verfügen und diese mit Hilfe von WINFOLine vertiefen bzw. erweitern wollen. Eine eigenständige Weitervermarktung an Lernende ist in beiden Fällen impliziert. In diesem Punkt ist die Erweiterung des Lehrveranstaltungsangebots an den Hochschulstandorten des WINFOLine-Kernkonsortiums zu nennen. Durch die gegenseitige Integration der WINFOLine-Lehrangebote sowie deren examenswirksame Anerkennung war es möglich, das bestehende Leistungsspektrum um jeweils 6 multimediale Veranstaltungen aufzustocken. Im Gesamten liefert WINFOLine einen Beitrag zur Stärkung der Fachdisziplin Wirtschaftsinformatik an den Partneruniversitäten. Für die Anspruchsgruppe der Studierenden entsteht durch das angereicherte Lehrveranstaltungsportfolio ein direkter Zusatznutzen durch die resultierende Attraktivitätssteigerung, die - im Sinne der Netzwerkökonomie - über den alleinigen Veranstaltungskatalog der jeweiligen Universität hinausgeht.

\section{Education-to-Business (E2B)- Abnehmer:}

Unternehmen, die Lehr-/Lerninhalte der Wirtschaftsinformatik als Bestandteil ihrer Personalentwicklungsstrategie ansehen und entsprechende Bildungsangebote und -dienstleistungen aufgrund von Externalisierungs-/ Outsourcingentscheidungen fremdbeziehen wollen. Die zur zielgerichteten Bedürfnisbefriedigung benötigten Lehr-/Lerninhalte und begleitenden Bildungsdienstleistungen (z.B. Betreuungsleistungen, technische Dienstleistungen, etc.) können aus den Angeboten des Bildungsnetzwerks zusammengestellt und mit Zertifikaten von Hochschulen versehen werden. Darüber hinaus können gemeinschaftlich definierte Anforderungen in kundenspezifische Lösungen umgesetzt werden. 


\section{Education-to-Customer (E2C)- Abnehmer:}

Privatpersonen, die sich im Bereich der Wirtschaftsinformatik wissenschaftlich weiterbilden und die Bildungsinhalte sowie ggf. eine Zertifizierung über das Bildungsnetzwerk WINFOLine beziehen wollen. Die Qualität und der Umfang der Bildungsleistung ist - unter Beachtung definierter Rahmenbedingungen (Anforderungen an Zertizierungen, z.B. Curricula) - individuell gestaltbar.

Das Bildungsnetzwerk WINFOLine fokussiert die dargestellten Bereiche mit unterschiedlichen, teils mit individuellen, aber auch mit mehrfach verwendbaren Dienstleistungsangeboten.

Im Bereich Education-to-Education existiert seit 1997 ein Tauschring, in welchem Bildungsangebote zwischen Hochschulen ausgetauscht werden. Die Tauschleistungen der Partner können immaterieller wie materieller Natur sein. Der Bereich Education-to-Business wird im Rahmen der Einbringung von Bildungsangeboten und -dienstleistungen aus dem Bildungsprodukt- und Servicepool in unternehmensspezifische Lernumgebungen realisiert, ferner finden die Bildungsangebote in unterschiedlichen Formen der kommerziellen Aus- und Weiterbildung Verwendung, so z.B. im Weiterbildungsstudiengang Informationsorganisation an der Universität Kassel. Der Bereich Education-to-Customer wird durch weitere verschiedenartige Weiterbildungsangebote wie bspw. einen vom Bildungsnetzwerk WINFOLine betriebenen Weiterbildungsstudiengang zum „Master of Science in Information Systems“" abgedeckt. Die partnerschaftliche Einrichtung weiterer Studiengänge an unterschiedlichen Institutionen des Aus- und Weiterbildungsmarktes ist in Planung.

Im Zentrum des Bildungsnetzwerkes WINFOLine steht der bereits erwähnte Bildungsdienstleistungspool. Dieser beinhaltet u.a. web-basierte Bildungsangebote unterschiedlicher Anbieter, die aufgrund ihrer modularen Struktur in Kombination mit abnehmerspezifisch festzulegenden Bildungsdienstleistungen, also z.B. Support- und Betreuungsleistungen, $\mathrm{zu}$ verschiedenartigen, zielgruppenkonformen Bildungsangeboten konfiguriert werden können.

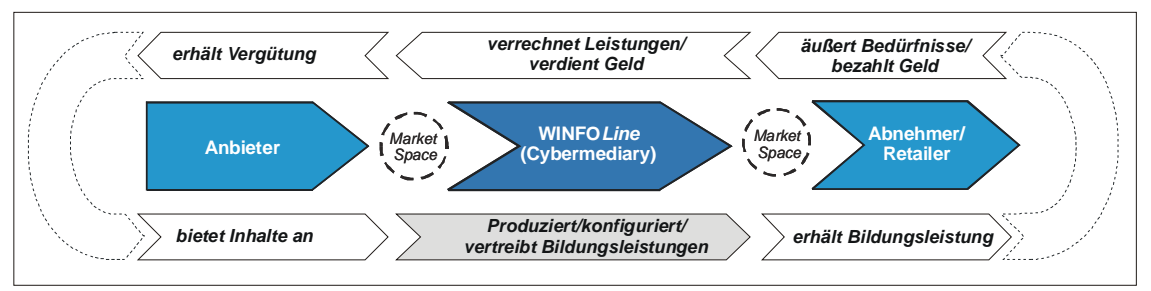

Abbildung 1a: Das Bildungsnetzwerk WINFOLine als Brokerinstanz

Wie Abbildung 1a aufzeigt fungiert das Bildungsnetzwerk als ein Broker im Sinne eines Cybermediary und koordiniert die Ebenen der Anbieter und Nachfrager 
(Bildungsbrokerage) nach Aus- und Weiterbildungsangeboten und -dienstleistungen. Die Rolle von Cybermediaries ist "... to select among various choices in the world. They act as gatekeepers, filtering and analyzing choices in order to help users reach a specific outcome. " [Boll96, S. 16].

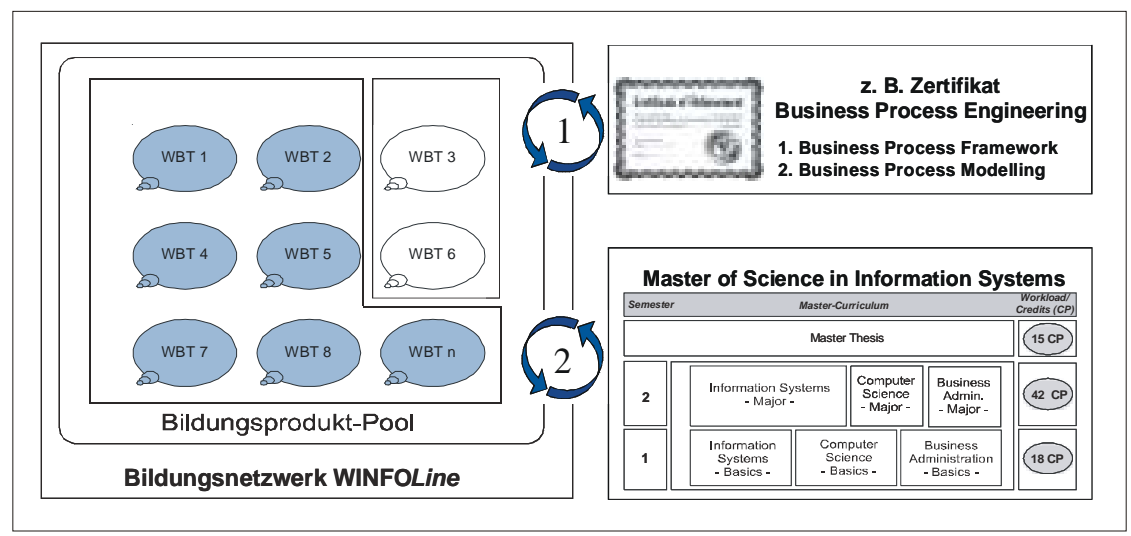

Abbildung 1b: Angebotskonfiguration im Bildungsnetzwerk WINFOLine

Abnehmer können sich aus den angebotenen Bildungsangeboten und dienstleistungen ein individuelles Bildungsangebot konfigurieren lassen. Abb. 1b verfeinert die abstrakte Wertkettenbetrachtung der virtuellen Brokerinstanz „WINFOLine“ (vgl. Abbildung 1a) im Punkt des unterlegen Prozesses der „Angebotsproduktion, -konfiguration, -vertrieb“. Gemäß der definierten kundenseitigen Ansprüche werden die im Bildungsproduktpool hinterlegten web-basierten Lehrinhalte zu einem neuartigen Angebot konfiguriert und dem Kunden offeriert. Die Reichweite des Angebotsspektrums vollzieht sich von der Bereitstellung einzelner Kurse mit Zertifizierungsmöglichkeit bis hin zu einem intregrierten Produktangebot, dem dargestellten universitären Weiterbildungsstudiengang „WINFOLine Master of Science in Information Systems“. In Abhängigkeit mit dem jeweiligen Abnehmersegment können die Bildungsangebote entweder direkt an Endabnehmer (Privatpersonen) oder an institutionelle Abnehmer vertreiben werden, die diese ihre bestehende Bildungsorganisation bzw. ihre Bildungsaktivitäten einzubinden oder als Retailer an weitere nachgelagerte Zielgruppen (Mitarbeiter, Studierende) weiterleiten. Abbildung 2 vedeutlicht die beschriebene Aufteilung als direktes (einstufiges) bzw. indirektes (mehrstufiges) Transaktionsmodell in Verbindung mit den fokussierten Abnehmersegmenten. Analog der absatzseitigen Betrachtung, erfolgen die Rückkopplungen entsprechend unmittelbar zwischen Endabnehmer und dem Bildungsnetzwerk WINFOLine oder mittelbar über die Einbeziehung definierter Ansprechpartner bei den institutionellen Abnehmern. Inhalt der Rückkopplung können einerseits die Neugestaltung von Bildungangeboten thematisieren, andererseits auch Verbesserungsvorschläge aus Nutzersicht beinhalten. Der rückläufige Interaktionsprozess leistet einen Beitrag für die Operationalierung des gegenseitigen Voneinander-Lernens im Sinne eines Continious 
Process Improvements sowie zur Identifikation evt. Auftretender Bedürfnisänderungen der Bildungsangebotsnachfrager (vgl. Kap. 2).

Losgelöst von der Mittelbarkeit der Transaktionsbeziehung eröffnet WINFOLine die Möglichkeit kundengesteuerte Angebote bereitzustellen, diskontinuierlich einsetzende Qualifikationsmaßnahmen durchzuführen bzw. das Vorhandensein unzureichender Qualifikationsangebote vor Ort auszugleichen [Kort98]. Das heißt, der Ressourcenproblematik des traditionellen hochschulischen Bildungswesens wird entgegengewirkt und die Angebote können aufgrund ihrer Modularität weitergehend (auch mehrfach) vermarktet werden.

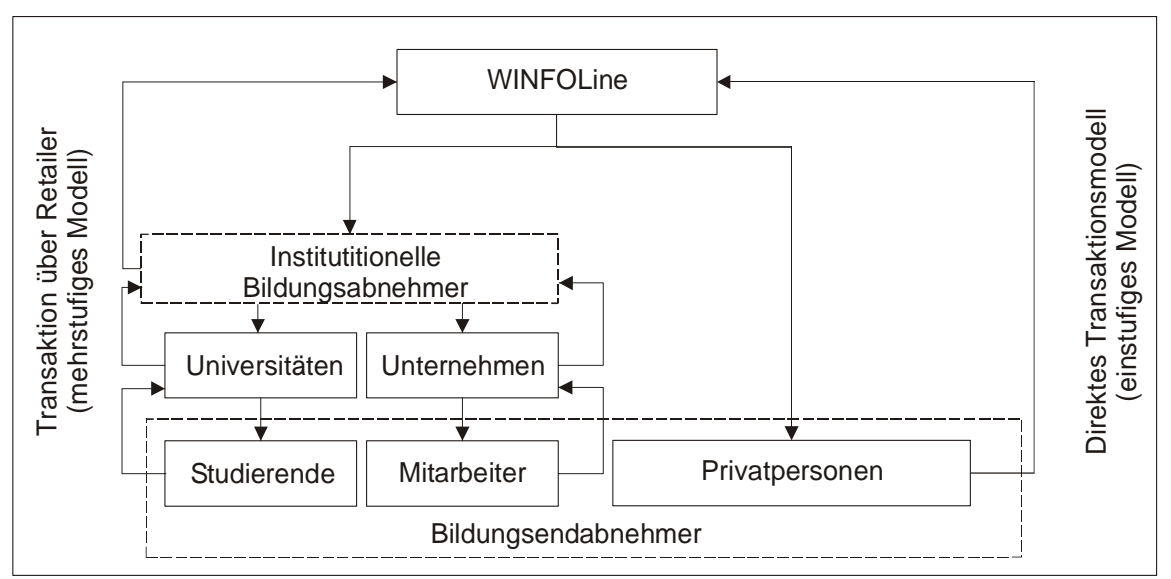

Abbildung 2: Transaktionsmodell des Bildungsnetzwerks WINFOLine

Das Angebot standardisierter Verrechnungs-, Finanzierungs-, Austausch- und Organisationsmodelle flankiert das Leistungsspektrum des Bildungsnetzwerks WINFOLine. Diese Dienstleistungen leisten einen Beitrag zur Schaffung marktorientierter Rahmenbedingungen zum Austausch der „Ware Bildung“ zwischen beliebig vielen Partnern.

\subsection{Vorteile web-basierter Bildungsangebote}

Ein Vorteil web-basierter Bildungsangebote und -dienstleistungen ist in der potenziellen Möglichkeit zur Individualisierung der Lehr-/Lernelemente zu sehen. Das Ziel einer Individualisierung der Kundenbeziehung wird mit Hilfe der virtualisierten Gestaltung von Bildungsangeboten verfolgt. Mit der Virtualisierung akademischer Wissensvermittlung wird u.a. die Intention verfolgt, einen Beitrag zur marktorientierten Gestaltung von Bildungsangeboten zu leisten und neue Geschäftsfelder und -erfolge zu generieren. Die gewählte Klassifizierung wird anhand der nachfolgend durch ein Bildungsprozess- und Bildungsproduktintelligenzportfolio dargestellten Adaption des Wissensintensitätsportfolios nach Porter/Millar veranschaulicht [PoMi85, S. 26f; ReKr96, 32f]. Die Einordnung erfolgt 
anhand der Einschätzung der Intelligenz des gewählten Vertriebsweges sowie der Intelligenz der Bildungsleistung.

Als intelligent gelten in diesem Kontext Systeme und Bildungsangebote, die eine hohe Flexibilität bzgl. der Ansprüche der beteiligten Parteien aufweisen und mit „Sense und Response“ [Kotl ${ }^{+} 02$, S. 13] gegenüber diesen ausgestattet sind, also die Möglichkeit zum „Customizing the Relationship“ und/oder „electronic customer care" in vollem Umfang zulassen [Hild98, S. 71].

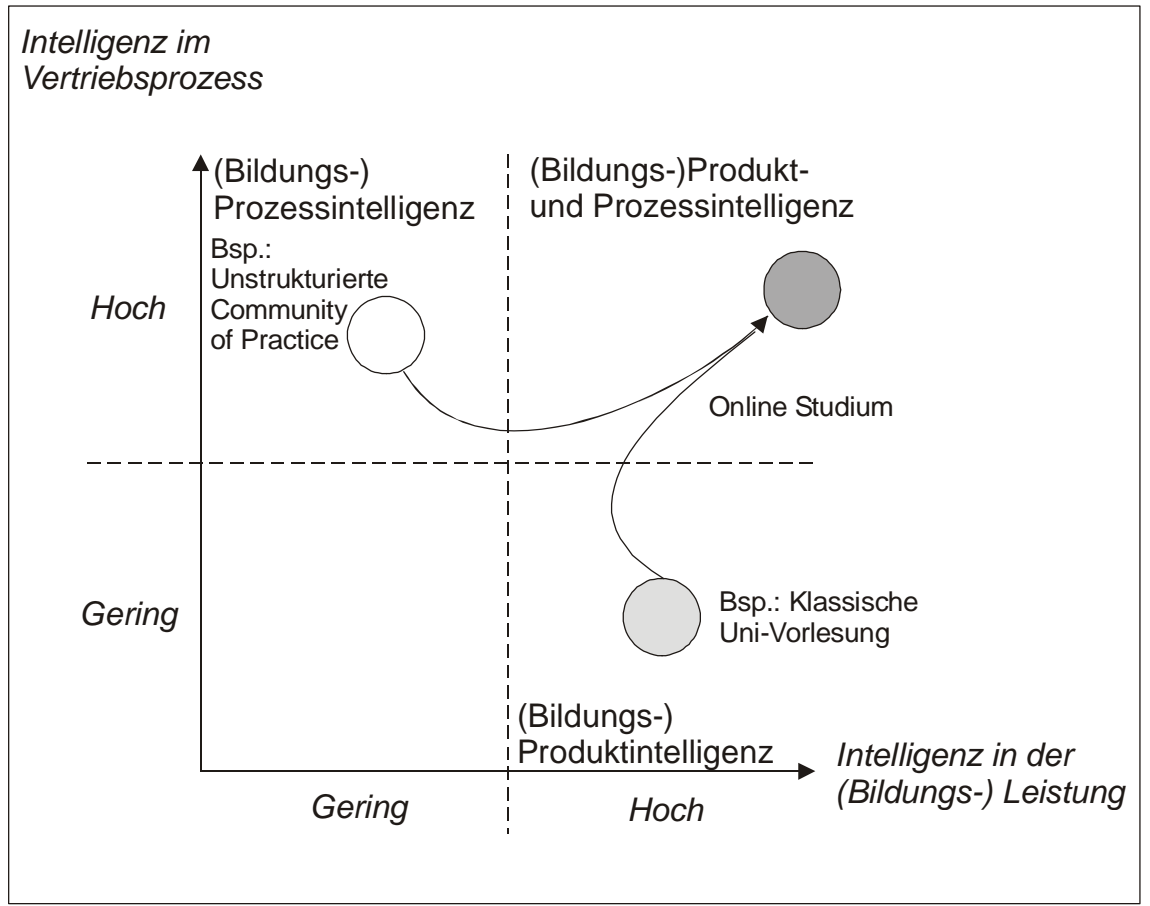

Abbildung 3: Bildungsprozess- und Bildungsproduktintelligenzportfolio [in Anlehnung an PoMi85, S. 27; ReKr96, S. 32f]

Die Abbildung 3 verdeutlicht, dass sich die Intelligenz bzgl. des Vertriebsprozesses beim originären akademischen Wissensvermittlungsprozess als niedrig darstellt, obwohl die Leistungen, und damit die Intelligenz in der Bildungsleistung, generell hoch einzuschätzen sind. Begründen lässt sich dies dadurch, dass der Vermittlungsprozess von qualitativ hochwertigen akademischen Inhalten durch klassische Präsenzveranstaltungen - aufgrund feststehender Zeitpunkte und Orte wenig Flexibilität aufweist.

Aus der für Vorlesungen charakteristischen Passivität der Leistungsempfänger heraus stellt sich die Gestaltung der Inhalte als Übertragung der Sichtweise des 
Lehrenden auf die Lernenden dar (In/Out-Sicht) [Wina ${ }^{+} 98$, S. 4]. Eine potenzielle Gefahr besteht darin, dass die vermittelten Inhalte eine weite Streubreite und somit bzgl. der Übereinstimmungen der Anspruchsgruppen eine geringe Treffsicherheit aufweisen. Das Interessen-Cluster bzw. die Wahlfreiheit spielt sich lediglich im Rahmen eines Korridors ab, der durch das vorhandene Lehrveranstaltungsangebot im Studiengang der Heimathochschule sowie die vorhandenen Ressourcen (Hörsäle etc.) beschränkt wird. Kapazitäts- und Angebotsrestriktionen wirken kontraproduktiv zu der von Seiten der Lernenden gewünschten Flexibilität. Interaktionspunkte zur Abstimmung der Ansprüche von Lernenden und Lehrenden werden lediglich sporadisch genutzt. Die niedrige Intelligenz in der Leistung unstrukturierter Communities of Practice im Sinne von Web-Communities wird durch die unstrukturierte Darstellung des Contents, den fehlenden redaktionellen Eingriff zur Vermeidung von Informationsredundanzen sowie die mangelnde Entfernung veralteter Informationen determiniert. Hohe Suchkosten und Frustration sowie ein „Information Overload“ können aus diesen Faktoren resultieren.

Web-basierte Angebote und Medien zur Informationsverbreitung ermöglichen hingegen den orts- und zeitpunktunabhängigen On-Demand-Zugriff. [HaJo94, S. 199]. Durch ihre Anreicherung mit intelligenten Suchtechnologien und synchronen Mensch-Maschine-Interaktionsystemen ergeben sich Möglichkeiten zur Lösung der diskutierten Problematik. Im Gegensatz zur Durchführung klassischer Präsenzveranstaltungen birgt der elektronische Medieneinsatz die Perspektive einer stärkeren, marktgerechten Verzahnung von Arbeits- und Lernphasen in sich [Eule92, S. 60]. Das web-basierte (Online-) Studium integriert die komplementären Stärken beider Systeme, unter weitgehender Vermeidung spezifischer Schwächen, in einem Gesamtangebot. Die qualitativ hochwertigen Inhalte akademischer Lehre werden mit Hilfe innovativer Infrastrukturen On-Demand zum Kunden/Lernenden geliefert. Diese Flexibilität reicht von der Mass-Customization von Bildungsangeboten, z.B. im Rahmen von Masterstudiengängen, bis hin zur bedürfnisorientierten Konfiguration von Bildungsmaßnahmen für die betriebliche Weiterbildung. 


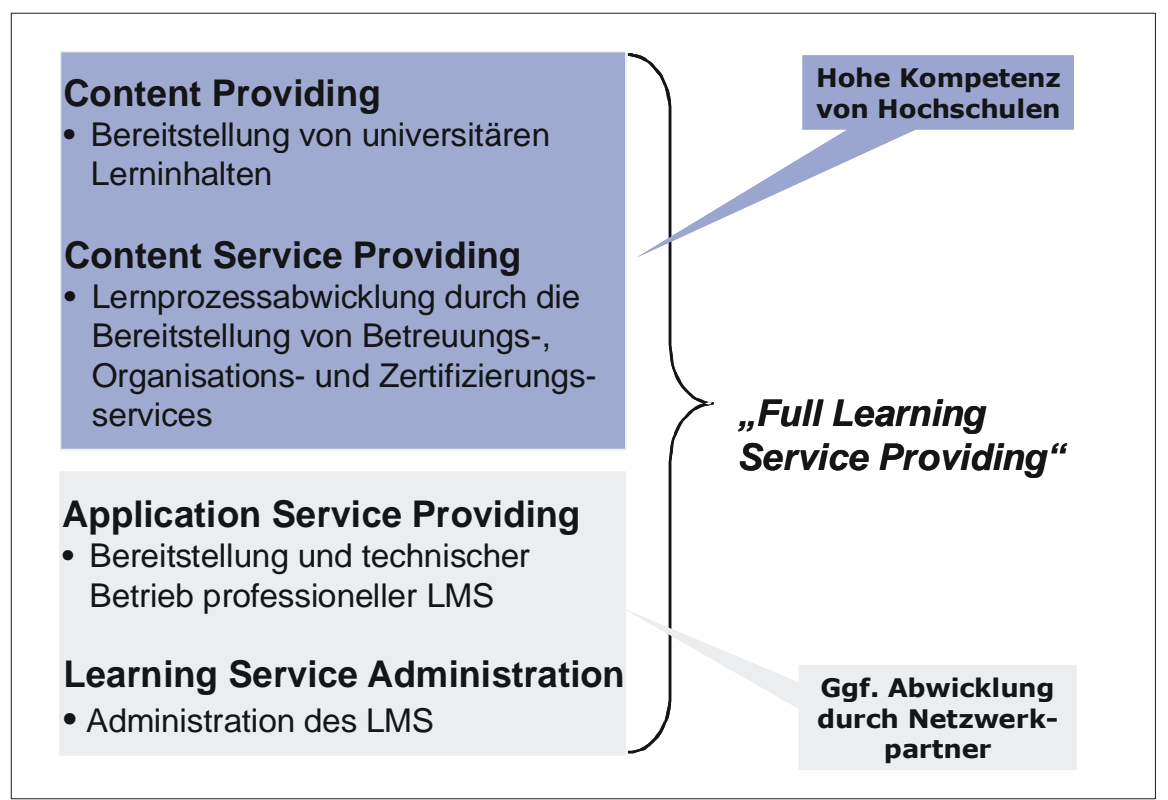

Abbildung 4: Angebotsportfolio im Kontext des Learning Service Providings

Durch die massiven Erfahrungen von Hochschulen in Lehre und Wissenschaft sowie den bestehenden Zertifizierungsmöglichkeiten bestehen Chancen für Hochschulen, sich als Learning Service Provider (LSP) auf dem eLearningBildungsmarkt zu positionieren. Das Angebot von LSP erstreckt sich potenziell über mehrere Bereiche, die in Abhängigkeit von den Zielkundensegmenten (Privatkunden, institutionelle Kunden) einzeln oder als Gesamtpakete bereitgestellt werden können [Krae 01, S. 207]. Bei der kompletten Lieferung aller in Abbildung 4 ausgewiesenen Leistungen aus einer Hand ist von Full-Learning-ServiceProviding zu sprechen. Kooperationen mit Partnern aus dem wissenschaftlichen (größtenteils öffentlichen) wie dem unternehmerischen Bereich sind in allen dargestellten Bereichen denkbar, zu analysieren und durchaus anzustreben. In einigen Bereichen erscheinen entsprechende Überlegungen besonders aus rechtlicher Sicht, aber auch aus ökonomischer Perspektive, dringend nötig. Weiterhin erscheint zur zielgruppengerechten, effizienten Erstellung und Nutzung von Bildungsangeboten und -dienstleistungen eine Konzentration und Fokussierung auf die Kernkompetenzen jeder leistungserbringenden Einheit unabdinglich. Diese Annahme definiert eine Herausforderung, welche sich auf die Profilbildung der Bildungsdienstleitungen positiv und wettbewerbsdifferenzierend auswirkt. 


\subsection{Praktische Erfahrungen im Bildungsnetzwerk WINFOLine}

Angereichert mit dem Netzwerkgedanken und der Konzentration der Leistungen anbietenden Partner auf ihre Kernkompetenzen können verteilte Koryphäen innerhalb von Angebotssystemen wie dem Bildungsnetzwerk WINFOLine effektiv zusammengeführt werden.

Der Mehrwert für die Abnehmer in Aus- und Weiterbildungsszenarien liegt in der Aktualität und Reichhaltigkeit der Inhalte sowie der Freiheit, aus einem breiten Spektrum an Bildungsangeboten und hochqualifizierten, begleitenden Dienstleistungen auswählen zu können. Sie profitieren von den vielfältigen Kompetenzen renommierter Lehrstuhlinhaber oder spezialisierter Dienstleister, die dem Bildungsnetzwerk angeschlossen sind (Best-of-Peer)

Der Output der gemeinschaftlichen Leistung im Rahmen eines Bildungsnetzwerkes ist höher einzuschätzen als die Summe der Einzelleistungen der beteiligten Partner.. Es lassen sich effiziente Aus- und Weiterbildungsszenarien aus bestehenden Lehr-/Lerninhalten generieren. Ein Beispiel hierfür stellt der an der Universität Göttingen etablierte Studiengang zum „Master of Science in Information Systems" dar.

\section{Fazit und Ausblick}

Die prognostizierten Entwicklungen auf dem Bildungsmarkt zeichnen ein positives Bild für die Vermarktung web-basierter akademischer Bildungsangebote in Szenarien der Aus- und Weiterbildung. Damit die staatlichen deutschen Hochschulen die bestehenden Marktchancen realisieren können, bedarf es in Teilen einer strategischen Neuausrichtung. Diese muss beinhalten, dass akademische Bildungsangebote nicht mehr ausschließlich aus Hochschul- und Hochschullehrersicht geplant werden, sondern sich ,zentral an den Erwartungen, Bedürfnissen, Wünschen und Qualitätsauffassungen des Kunden“ [KoB195, S. 66] orientieren. Die Hochschulen müssen lernen, Bildungsnachfrager zu identifizieren, Wissen über diese aufzubauen und sie mit kundenorientierten, individualisierten Bildungsangeboten zu versorgen [Sche00, S.183ff]. Dies gilt gleichermaßen für den Prozess der hochschulischen Ausbildung wie auch für die Phasen der inner- und außerbetrieblichen Weiterbildung. Aus Effizienz- und Wettbewerbsgründen sind hierfür zukünftig notwendige Kernkompetenzen innerhalb der Hochschulen zu definieren und ggf. auszubauen.

Zu diesem Zweck sind auch hochschulseitig Strategien zu entwickeln, die mit Hilfe flexibler Organisationsstrukturen und innovativer Technologien in intelligente und alltagstaugliche Lösungen umgesetzt werden können und den individuellen 
Ansprüchen der Abnehmer entsprechen $\left[\mathrm{Bohl}^{+} 02 \mathrm{a}\right]$. Erfahrungen beim Aufbau virtueller Bildungsangebote von WINFOLine zeigen, dass qualitativ hochwertige eLearning-Angebote in (Hochschul-) Verbünden, Partnerschaften bzw. sonstigen Kooperationen effektiv und marktgerecht erstellt werden können[Bohl $\left.{ }^{+} 02 \mathrm{~b}\right]$. Dabei können Kooperationen in und zwischen Hochschulen, aber auch zwischen Leistungserbringern aus Wissenschaft und Unternehmen hilfreich sein. Ein permanenter Vergleich mit privatwirtschaftlichen und öffentlichen Anbietern von in den Bildungsangeboten integrierten Dienstleistungen und die Analyse möglicher Kooperationspartner erscheint aus Effizienzgründen unabdingbar.

\section{Literatur}

[BeHu01] Bentlage, U./ Hummel, J.: Märkte in den USA und in Deutschland im Vergleich. In: Bentlage, U./ Glotz, P./ Hamm, I./ Hummel, J. (Hrsg.): E-Learning. Verlag Bertelsmann Stiftung. Gütersloh 2001, S. 121-153.

[Bohl'02a] Bohl, O./ Grohmann, G./ Martin, G.: Case-Study: Educational Network WINFOLine. In: Wagner, E.; Szucs; András (Hrsg.): Open and Distance Learning in Europe an Beyond - Rethinking International Cooperation. Proceedings of the 2002 EDEN Annual Conference. EDEN: Granada 2002, S. 511-513.

[Bohl'02b] Bohl, O./ Winand, U./ Grohmann, G./ Scheer, A.-W.: Virtuelle Bildungsnetzwerke: Struktur- und Betreibermodelle am Beispiel WINFOLine. In: Engelien, M.; Homann, J. (Hrsg.): Virtuelle Organisation und Neue Medien 2002. Eul: Lohmar, Köln 2002, S. 41-68.

[Bol196] Bollier, D.: The Future of Electronic Commerce. The Aspen Institute: Washington 1996.

[Del191] Dellmann, K.: Einflußgrößen der Erfolgsdynamik. In: Kistner, K. P.; Schmidt, R. (Hrsg.): Unternehmensdynamik-Festschrift zum 60. Geburtstag von Horst Albach. Wiesbaden 1991.

[Druc95] Drucker, P. (o.T.): In: Kotler, P./ Bliemel, F.: Marketing Management: Analyse, Planung, Umsetzung und Steuerung, 8. vollst. neu bearb. und erw. Aufl., Schäffer Poeschel: Stuttgart 1995.

[Ehre $\left.{ }^{+} 01\right]$ Ehrenberg, D./ Scheer, A.-W./ Schumann, M./ Winand, U.: Implementierung von interuniversitären Lehr- und Lernkooperationen: Das Fallbeispiel WINFOLine. In: Wirtschaftsinformatik 43. 2001. S. 5-11.

[Eule92] Euler, D.: Didaktik des computerunterstütztem Lernens: Praktische Gestaltung und theoretische Grundlagen. BW Bildung und Wissen: Nürnberg 1992.

[GrMa02] Grohmann, G./ Martin, G.: Ansatzpunkte zur Organisation virtueller Lernszenarien am Beispiel des Bildungsnetzwerkes WINFOLine. In: Schubert, S./ Reusch, B./ Jesse, N. (Hrsg.): Lecture Notes in Informatics (LNI) - Proceedings: Informatik be- 
wegt. Informatik 2002 - 32. Jahrestagung der Gesellschaft für Informatik e.V. (GI). 2002. Köllen: Bonn 2002, S. 319-324.

[HaJo94] O’Hara-Devereaux, M./ Johansen, R.: Global Work. Bridging Distance, Culture and Time. Jossey-Bass: San Francisco 1994.

[Hild98] Hildebrand, V. G.: Kundenbindung mit Online Marketing. In: Link, J. (Hrsg.): Wettbewerbsvorteile durch Online Marketing - Die strategischen Perspektiven elektronischer Märkte. Springer: Berlin et al. 1998, S. 53-75.

[HuEn02] Hutzschenreuter, T./ Enders, A.: Gestaltung internetbasierter Studienangebote im Markt für Managementbildung. In: zfbf - Schmalenbachs Zeitschrift für betriebswirtschaftlche Forschung. Jg. 54. September 2002. S. 543-561.

[Kort98] Kortzfleisch, H.F.O. v.: Virtualisierung der betrieblichen Aus- und Weiterbildung, Arbeitsbericht Nr. 22, Universität-Gh-Kassel, Fachgebiet Wirtschaftsinformatik, Kassel 1998.

[KoB195] Kotler, P./ Bliemel, F.: Marketing Management: Analyse, Planung, Umsetzung und Steuerung, 8. vollst. neu bearb. und erw. Aufl., Schäffer Poeschel: Stuttgart 1995.

[Kotl ${ }^{+}$02] Kotler, P./ Jain, D.C./ Maesincee, S.: Marketing der Zukunft: Mit Sense and Response zu mehr Wachstum und Gewinn. Campus: Frankfurt/Main, New York 2002.

$\left[\mathrm{Krae}^{+} 01\right]$ Kraemer, W./ Sprenger, , P./ Wachter, C.: Learning Services als Bestandteil einer eHR-Strategie. In: Scheer, A.-W. (Hrsg.): Die eTransformation beginnt! Lessons Learned, Branchenperspektiven, Hybrid Economy, M-Business. Physica: Heidelberg 2001, S. 191-228.

[Krae99] Kraemer, W.: Education Brokerage - Wissensallianzen zwischen Hochschulen und Unternehmen. In: Information Management \& Consulting, 14 Jg., Nr. 1, S. 17-26.

[Port97] Porter, M.E.: Wettbewerbsstrategie: Methoden zur Analyse von Branchen und Konkurrenten (Competitive Strategy). 9. Aufl., Campus: Frankfurt/Main 1997.

[PoMi85] Porter, M.E./ Millar, V.E.: How information gives you competitive advantage. In: North, K.: Wissensorientierte Unternehmensführung: Wertschöpfung durch Wissen, 2. aktualisierte u. erw. Aufl., Gabler: Wiesbaden 1999.

[ReKr96] Rehäuser, J./ Krcmar, H.: Wissensmanagement im Unternehmen. In: Schreyögg, G./ Conrad, P. (Hrsg.): Wissensmanagement. Managementforschung 6. DeGruyter: Berlin 1996.

[Riek95] Rieker, S.A.: Bedeutende Kunden. Gabler: Wiesbaden 1995.

[Schee00] Scheer, A.-W.: Unternehmen gründen ist nicht schwer... . Springer: Berlin, et al. 2000 .

[Wina ${ }^{+}$98] Winand, U./ Kortzfleisch, H.F.O. v.; Heller, U.: Electronic Learning Commerce, Arbeitsbericht 21. Universität-Gh-Kassel, Fachgebiet Wirtschaftsinformatik, Kassel 1998.

[Wina ${ }^{+}$96] Winand, U/ Kortzfleisch, H.F.O. v./ Pohl, W.: Online Aus- und Weiterbildung: Die Virtualisierung der Wissensvermittlung und des Lernens. Arbeitsbericht 7. Universität-Gh-Kassel, Fachgebiet Wirtschaftsinformatik, Kassel 1996. 